\section{$\overbrace{\substack{\text { CHEST } \\ \text { CUNIC }}}$}

${ }^{1}$ Pleural Disease Unit, Queen Elizabeth University Hospital, Glasgow, UK ${ }^{2}$ Institute of Infection, Immunity \& Inflammation, University of Glasgow, Glasgow, UK

\section{Correspondence to}

Dr Varun Sharma, Department of Respiratory Medicine, Queen Elizabeth University Hospital, 1345 Govan Road, Glasgow G51 4TF, UK; vsharma3@nhs. net

Received 1 May 2017

Revised 15 July 2017 Accepted 7 August 2017

Published Online First 28 August 2017

\section{CrossMark}

To cite: Sharma V, Blyth KG. Thorax 2018;73:91-93.

\title{
Empyema necessitans and a persistent air leak associated with rupture of an anaerobic lung abscess due to bacteroides
}

\author{
Varun Sharma, ${ }^{1}$ Kevin G Blyth ${ }^{1,2}$
}

\section{INTRODUCTION}

Here we report an unusual case of ruptured lung abscess, complicated by both a persistent air leak and empyema necessitans. This combination of problems, in a patient with significant co-morbidities presented major diagnostic difficulties and challenging pleural intervention issues. The case based discussion presented also highlights a number of important learning points that can be generalised to the assessment and management of patients with severe pleuro-pulmonary infections, including the minority associated with an acute air-leak.

\section{CASE BASED DISCUSSIONS}

Varun Sharma (VS): A morbidly obese $(120 \mathrm{~kg}$, body mass index 49) 43-year-old female presented to the Queen Elizabeth University Hospital Glasgow, obtunded and in acute respiratory distress. She had been bed or chairbound for 2 years due to chronic pain, primarily related to severe hidradenitis suppurativa (HS). Other past medical history included poorly controlled Insulin-dependent type 2 diabetes mellitus (glycated haemoglobin (HbA1c) level $107 \mathrm{mmol} / \mathrm{mol}$ on admission), asthma and non-alcoholic fatty liver disease. She reported a cough productive of large volumes of purulent, foul-smelling sputum and severe pain, particularly localised to the axillae and lateral chest walls. The patient was tachycardic (147 bpm), tachypnoeic and hypoxic. Oxygen saturations were recorded at $85 \%$ on air. Examination revealed accessory muscle use, reduced air entry, dullness to percussion and bronchial breathing at the right lung base. The patient did not tolerate a full skin examination due to pain, and reported multiple drug allergies, including suxamethonium and metronidazole, but no penicillin allergy. Treatment for probable community-acquired pneumonia was initiated (intravenous amoxicillin and oral clarithromycin) pending chest radiography. Initial laboratory investigations revealed a $\mathrm{C}$ reactive protein level over $300 \mathrm{mg} / \mathrm{L}$ and marked hypoalbuminaemia (14 g/L). Over the next few hours, the patient reported facial swelling. On repeat clinical assessment, no stridor or airway compromise was noted. Antibiotic allergy was suspected, and intravenous hydrocortisone and chlorphenamine were administered. However, her facial swelling worsened and a chest radiograph, acquisition of which has been delayed, revealed significant subcutaneous emphysema, right-sided pleural effusion, right lower zone consolidation and a small left apical pneumothorax. A further repeat physical examination (6-8 hours post-admission) revealed classical features of subcutaneous emphysema, which had been absent on admission. Oxygen requirements increased to $60 \%$ and the patient was transferred to the Medical High Dependency Unit (MHDU). Her antibiotics were changed to intravenous co-amoxiclav and oral clindamycin. A more comprehensive skin examination revealed multiple tender, inflamed skin lesions in the axillae and on the lateral aspects of the chest wall bilaterally, many of which were concealed beneath folds of adipose tissue. CT scanning of thorax and abdomen was requested and a surgical opinion. The differential diagnosis at this time was recorded as Boerhaave's syndrome, pneumonia or necrotising fasciitis secondary to HS complicated by a pleurocutaneous fistula.

Kevin G Blyth (KGB): The clinical presentation is most consistent with acute bacterial pneumonia with increasing oxygen requirements related to this. However, the development of rapidly evolving subcutaneous emphysema is highly concerning and must indicate rupture of a gas-containing structure in the thorax. Transfer to MHDU is entirely appropriate and should include an early discussion with the patient, her family and critical care colleagues regarding the ceiling of care given her extensive comorbidities and poor functional status. Boerhaave's syndrome was first reported in 1724 and describes acute, spontaneous, oesophageal rupture. The term 'spontaneous' differentiates Boerhaave's from iatrogenic oesophageal rupture, which accounts for $85 \%-90 \%$ of cases and both are associated with high mortality. ${ }^{1}$ However, Boerhaave's syndrome typically includes a history of significant prior vomiting. In addition, the majority of adults with Boerhaave's present with left-sided pleuropulmonary complications, ${ }^{1}$ including (hydro-) pneumothorax, pneumomediastinum, subcutaneous emphysema and pneumonia. The current case is not typical of Boerhaave's, since the clinical findings appear bilateral and predominantly right sided, and there is no history of prior vomiting. Necrotising fasciitis complicating HS appears very unlikely, given the absence of any skin defects associated with a visible or audible air leak.

VS: CT imaging reported right lower lobe consolidation, an adjacent right hydropneumothorax and a left apical pneumothorax (see figure $1 \mathrm{~A}$ ). The upper lobe of the right lung was fully inflated. Extensive subcutaneous emphysema 


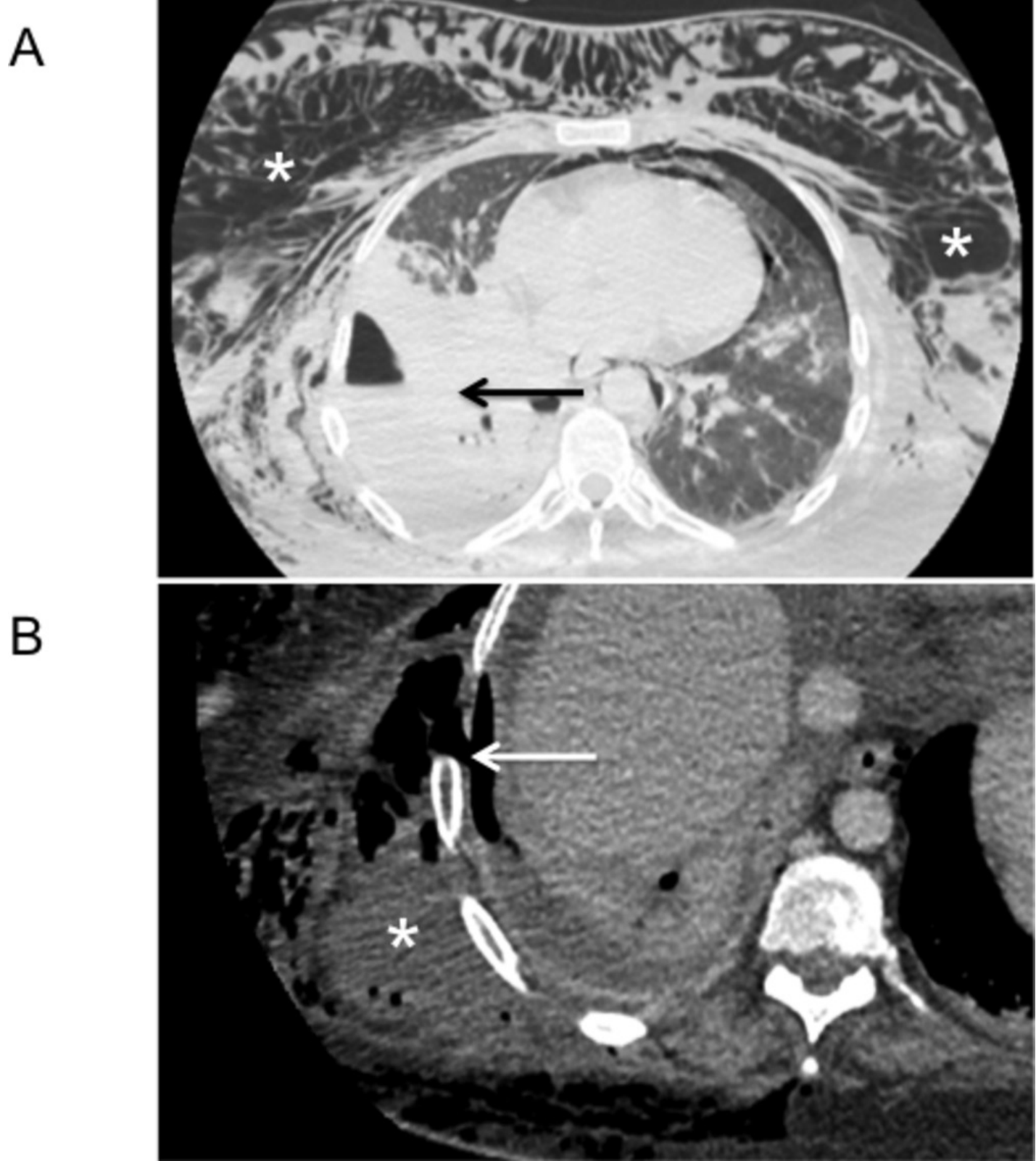

Figure 1 CT images (lung settings (A), mediastinal settings (B) acquired in a female patient with empyema necessitans (EN) and a persistent air leak related to a ruptured right lower lobe lung abscess. Extensive subcutaneous emphysema is highlighted in panel A (white asterix), which also shows right lower lobe lung consolidation (black arrow), an adjacent hydropneumothorax, a small left pneumothorax and a pneumomediastinum. Panel B shows a defect in the parietal pleura and subpleural tissues (white arrow), resulting in discharge of infected pleural fluid into the subcutaneous tissues (EN, white asterix).

was confirmed on both sides of the chest, extending into the neck and associated with pneumomediastinum. A gastrograffin swallow 24 hours after MHDU admission failed to identity an oesophageal leak, making Boerhaave's syndrome unlikely. With the diagnosis uncertain and the patient's subcutaneous emphysema worsening, a left apical $12 \mathrm{~F}$ intercostal drain (ICD) was sited under CT guidance on day 3 of the admission. A referral was made to the pleural disease team on day 4 .

KGB: At first review on day 4, the patient was tachypnoeic and in pain, despite strong opiate analgesia. Intravenous sedation was being used for distress related to expanding subcutaneous emphysema, which was now precluding eye-opening and verbal communication. Oxygen saturations were $94 \%$ on a non-rebreathing trauma mask. The left-sided ICD was swinging but not bubbling, and no significant volume of pleural fluid had been drained. The two recent CT scans were reviewed. The second CT (acquired during left-sided drain insertion) revealed an unreported new defect in the lateral surface of the parietal pleura and a modest increase in the size of the right effusion (see figure $1 \mathrm{~b}$ ). A diagnosis of empyema necessitans (EN) was made, likely associated with a ruptured right lower lobe lung abscess. Incidentally, a risk factor supporting this is the patient's poor diabetic control. Given the declining clinical situation, immediate insertion of a large bore ICD was deemed necessary for air-leak control. However, the patient's body habitus, the extent of her HS and subcutaenous emphysema suggested that this would be technically challenging, and would require additional sedation. The risks associated with this were explained to the patient and her 
husband, who provided informed written consent. Intensive care agreed to admit the patient if her respiratory function deteriorated further during large bore drain insertion. The patient was placed into the left lateral decubitus position and sedated using bolused intravenous midazolam (13 $\mathrm{mg}$ in total over $50 \mathrm{~min}$ ). After extensive blunt dissection, the parietal pleura was eventually opened and a $24 \mathrm{~F}$ ICD placed to the right apex. Thoracic ultrasound could not be used because of the subcutaneous emphysema. The drain was attached to a digital chest drainage unit (Thopaz, Medela, Switzerland), which recorded an initial airflow rate of $1300 \mathrm{~mL} / \mathrm{min}$. Five hundred and fifty millilitres of purulent pleural fluid was drained over the next few days.

VS: The patient's respiratory function improved within the first few hours and endotracheal intubation was not required. Pleural fluid culture results demonstrated Bacteroides species. Digital airflow readings gradually reduced over the subsequent 10 days and both pneumothoraces and the subcutaneous emphysema resolved. The $24 \mathrm{~F}$ right ICD was removed after three consecutive days of no measurable air leak. The patient was discharged home on day 16 and completed 6 weeks of antibiotic therapy (co-amoxiclav). At pleural clinic review, 3 months later her chest radiograph had almost returned to normal.

\section{DISCUSSION}

VS: EN occurs when infected pleural fluid dissects the parietal pleura and invades the subpleural tissues. This facilitates discharge of pleural contents (fluid \pm air if there is an associated viscero-pleural fistula) into the subcutaneous tissues. In severe cases, this discharge may be externalised. ${ }^{2}$ EN usually results from inadequately treated intrapulmonary or pleural infection. It is therefore now a rare clinical entity, given high levels of awareness regarding the importance of early treatment of sepsis and early drainage of pleural infection. Classically, patients with EN present with severe sepsis and an acutely tender chest wall mass, usually on a background of preceding respiratory symptoms. EN lesions are said to be more common on anterior thoracic surfaces, where the parietal pleura is less adherent to the lung. ${ }^{3}$ Interestingly, previous authors have reported misdiagnosis of EN as HS, ${ }^{2}$ which was a major confounding factor in the case reported here. In this case, the patient's body habitus, her preceding history of chronic pain and the extent of her subcutaneous emphysema confounded the initial clinical assessments made. As a result, EN did not feature in the initial differential diagnosis made, which may have been influenced by the reporting of the emergency CT scans which failed to identify the pathognomonic chest wall defect.

KGB: This case highlights a number of important learning points. The first is that EN, although rare, should be actively considered in patients presenting late with severe pulmonary or pleural infection, particularly if severe chest pain is reported. Second, earlier review by a respiratory specialist with experience in complex pleural disease may have led to a less fraught definitive drainage procedure than was ultimately necessary. Specifically, an early although technically challenging diagnostic thoracentesis from the right pleural space should have been attempted and may have revealed pleural infection, prompting earlier definitive drainage. As suggested earlier, the clinical presentation was not typical for Boerhaave's syndrome, which is frequently associated with dissection of gastrointestinal gas into the left pleural space. This misdiagnosis led directly to the left pleural space being incorrectly identified as the likely source of the air leak, delaying definitive (right-sided) pleural intervention. The left-sided pneumothorax and the pneumomediastium were probably caused by pressurised air tracking from the leaking right lower lobe through the pulmonary ligament and/ or directly into the mediastinum via additional parietal pleural defects not visible on CT. Third, the pathogen identified in this case (Bacteroides) is a relatively uncommon cause of EN, which is most frequently caused by Mycobacterium tuberculosis and less commonly by Actinomyces israelii, Staphylococcus aureus, Streptococcus milleri, Mycobacterium avium-intracellulare and Aspergillus fumigatus. ${ }^{4}$ However, Bacteroides is a common cause of intrapulmonary abscess and pleural infection, which were the primary disease mechanisms here. ${ }^{5}$ Finally, the case highlights the management principles for all cases of $\mathrm{EN}$, which include appropriate antibiotic therapy and control of the pleural space, including air-leak management where necessary, facilitating lung re-expansion and closure of any viscero-pleural fistula.

Competing interests None declared.

\section{Patient consent Obtained.}

Provenance and peer review Not commissioned; externally peer reviewed.

(C) Article author(s) (or their employer(s) unless otherwise stated in the text of the article) 2018. All rights reserved. No commercial use is permitted unless otherwise expressly granted.

\section{REFERENCES}

1 Hingston CD, Saayman AG, Frost PJ, et al. Boerhaave's syndrome - rapidly evolving pleural effusion; a radiographic clue. Minerva Anestesiol 2010;76:865-7.

2 Magness DJ. Empyema necessitans caused by mycobacterium tuberculosis in an immunocompetent patient. WMJ 2013;112:129-30.

3 Arora A, Rajesh S, Patidar Y. Empyema necessitatis: yet another mimic of necrotizing fasciitis in the torso. AJR Am J Roentgenol 2015;204:W734-5.

4 Rendon A, Rendon RA, Bauerle O. Empyema necessitatis: unique presentation in a coccidioidomycosis case. Am J Respir Crit Care Med 2015;191:964-6.

5 Maskell NA, Batt S, Hedley EL, et al. The bacteriology of pleural infection by genetic and standard methods and its mortality significance. Am J Respir Crit Care Med 2006;174:817-23 


\section{Correction: Empyema necessitans and a persistent air leak associated with rupture of an anaerobic lung abscess due to bacteroides}

Sharma V, Blyth KG. Empyema necessitans and a persistent air leak associated with rupture of an anaerobic lung abscess due to bacteroides. Thorax 2018;73:91-3. doi: 10.1136/ thoraxjnl-2017-210462.

Figure 1 of this paper was published in print with the colours inverted. This makes the annotation of the figures (black/white arrow, black/white asterix) stated in the figure legend incorrect. Please find the correct figure and legend below.

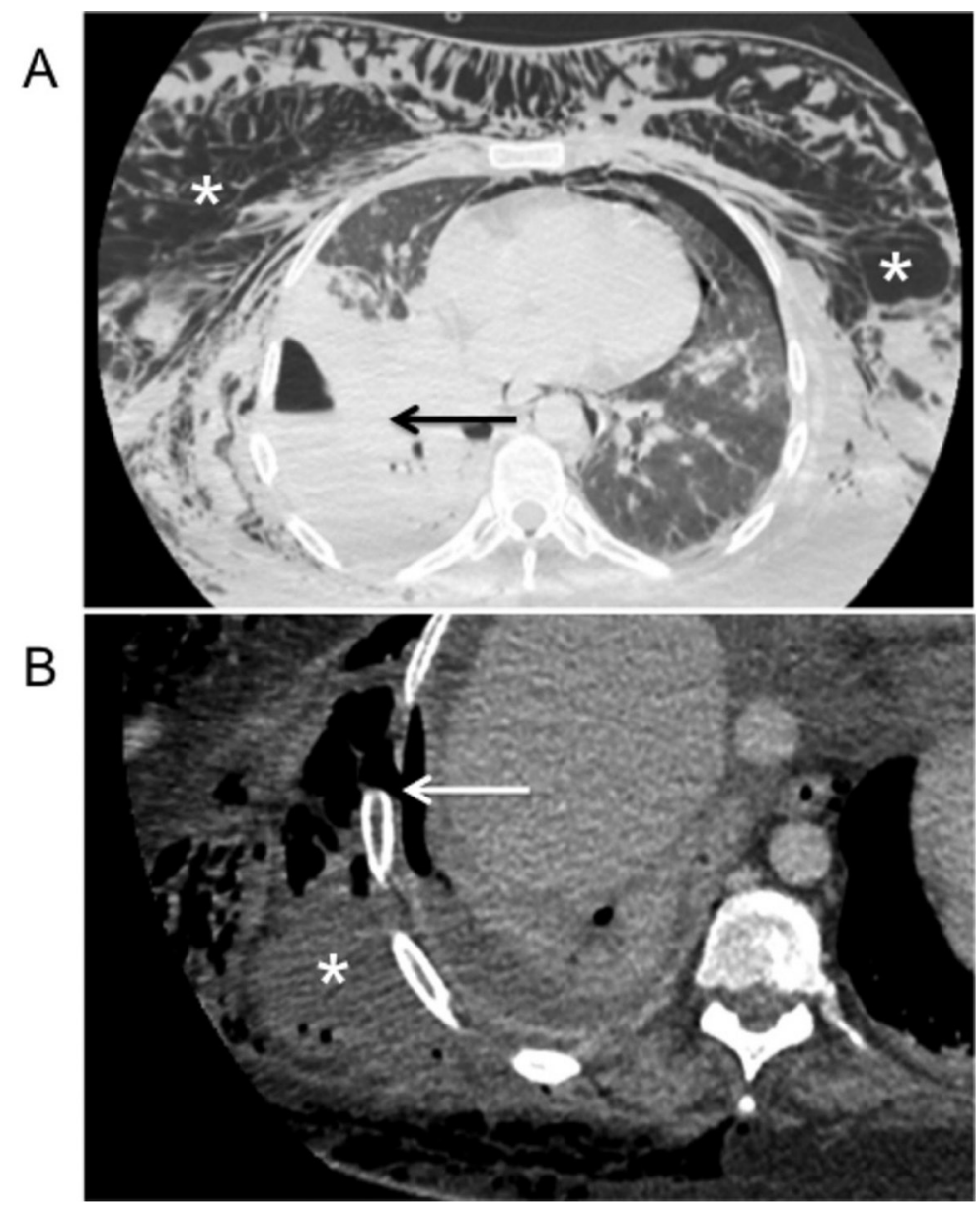

Figure 1 CT images (lung settings (A), mediastinal settings (B) acquired in a female patient with empyema necessitans (EN) and a persistent air leak related to a ruptured right lower lobe lung abscess. Extensive subcutaneous emphysema is highlighted in panel A (white asterix), which also shows right lower lobe lung consolidation (black arrow), an adjacent hydropneumothorax, a small left pneumothorax and a pneumomediastinum. Panel B shows a defect in the parietal pleura and subpleural tissues (white arrow), resulting in discharge of infected pleural fluid into the subcutaneous tissues (EN, white asterix).

(C) Article author(s) (or their employer(s) unless otherwise stated in the text of the article) 2018. All rights reserved. No commercial use is permitted unless otherwise expressly granted.

Thorax 2018;73:404. doi:10.1136/thoraxjnl-2017-210462corr1

(D) Check for updates 